\title{
Dealing with Renin-Angiotensin Inhibitors, Don't Mind Serum Creatinine
}

\author{
Piero Ruggenentia, ${ }^{a}$ Giuseppe Remuzzi ${ }^{a}$, b \\ ${ }^{a}$ Mario Negri Institute for Pharmacological Research, Clinical Research Center for Rare Diseases - \\ Aldo e Cele Daccò, Villa Camozzi, Ranica, and b Unit of Nephrology, Azienda Ospedaliera Ospedali Riuniti di \\ Bergamo, Bergamo, Italy
}

In 1997, Apperloo et al. [1] reported that in 40 patients with non-diabetic chronic kidney disease (CKD), antihypertensive treatment was associated with a short-term glomerular filtration rate (GFR) fall that inversely correlated with subsequent GFR decline. Patients with a larger fall eventually had a slower decline and even a GFR stabilization in some cases, whereas those with smaller or no short-term changes had the fastest renal function loss in the long run. Since the GFR fall was associated with a reduction in filtration fraction and fully recovered after treatment withdrawal, the authors guessed that their findings could be explained by a treatment-induced reduction of glomerular intracapillary pressure. Based on previous evidence that glomerular pressure reduction limited proteinuria and glomerulosclerosis in several experimental models of diabetic and non-diabetic CKD [2], the authors hypothesized that a similar mechanism could explain the long-term renoprotective effect of antihypertensive treatment observed in their patients.

In the following years, evidence accumulated that GFR and serum creatinine changes associated with blood pressure (BP) reduction are especially prominent in subjects with a long-lasting markedly elevated $\mathrm{BP}$ and renal insufficiency, in particular when the antihypertensive regimen includes inhibitors of the renin-angiotensin sys- tem (RAS) such as angiotensin-converting enzyme inhibitors (ACEi) or angiotensin II receptor blockers (ARBs). A review of 1,102 CKD patients from 12 randomized clinical trials clearly showed that in these cases the initial serum creatinine increase associated with RAS inhibitor-based antihypertensive regimens is also followed by slower disease progression [3]. Note that larger shortterm increases were associated with slower GFR decline in the long term. Thus, as originally suggested by Apperloo et al. [1], short-term serum creatinine increases associated with BP reduction 'should be rewarded as an evidence of efficacy rather than an unwanted effect of treatment'. Despite the above findings, however, the very authors of the above review suggested that when serum creatinine increases exceed $30 \%$ within the first 2 months of RAS inhibition, treatment should be withdrawn [3]. This recommendation was also noted in the National Kidney Foundation K/DOQI clinical guidelines [4]. However, neither the review nor the K/DOQI guidelines provided any evidence that short-term serum creatinine increases exceeding the above threshold are associated with long-term accelerated renal function loss.

In this issue of the American Journal of Nephrology, Hirsch et al. [5] strongly suggest that the opposite may be true regarding initial changes in serum creatinine and

\section{KARGER}

Fax +4161306 1234 E-Mail karger@karger.ch www.karger.com
(C) 2012 S. Karger AG, Basel

0250-8095/12/0365-0427\$38.00/0

Accessible online at:

www.karger.com/ajn
Giuseppe Remuzzi, MD, FRCP

Mario Negri Institute for Pharmacological Research, Centro Anna Maria Astori

Science and Technology Park Kilometro Rosso

Via Stezzano, 87, IT-24126 Bergamo (Italy)

E-Mail giuseppe.remuzzi@marionegri.it 
outcome. The authors described the outcome of $48 \mathrm{pa}$ tients with proteinuric CKD, mostly African-Americans who had been treated for at least 3.5 years with ACEi and/ or ARBs at doses that, independent of changes in serum creatinine, targeted a systolic BP of $\leq 125 \mathrm{~mm} \mathrm{Hg}$ and a urinary protein $\leq 30 \mathrm{mg} / \mathrm{dl}$. Serum creatinine increased in 40 patients and the increase exceeded $30 \%$ in 20 cases. Treatment was well tolerated in all patients, including those with larger serum creatinine increases. Of interest, the rate of estimated GFR decline during the whole observation period was more than halved in patients with serum creatinine increases exceeding $30 \%$ as compared to those with smaller or no changes.

The authors found that slower progression in subjects with larger creatinine increases was associated with more frequent use of furosemide and more effective proteinuria reduction, whereas BP control was similar between groups. This confirms previous evidence that GFR- and proteinuria-reducing effects of RAS inhibition are both enhanced by sodium depletion, which in turn may contribute to maximize renoprotection in this context [6]. The higher prevalence of females among patients with a larger treatment effect might reflect better compliance, higher body-weight-adjusted dosages of study medications, or some gender-related confounding factor.

The findings of this study, however, must be taken with caution because of the retrospective and uncontrolled design and the small sample size. It is noteworthy that the present findings confirm and extend results of a previous prospective, matched-cohort study evaluating the effect of a multidrug treatment titrated to urinary proteins - the Remission Clinic program - in 112 CKD subjects at high risk of progression to ESRD because of persistent nephrotic range proteinuria despite treatment with standard antihypertensive dosages of an ACEi [7]. In study patients, multidrug treatment, including dual RAS blockade with ACEi and ARBs up-titrated to maximum tolerated doses independent of changes in serum creatinine levels, was associated with larger proteinuria reduction and remarkably slower GFR decline than in historical reference patients on a conventional regimen titrated to BP control. During the 7-year observation, only 2 of the 56 patients who were treated according to the Remission Clinic protocol progressed to ESRD compared to 17 of the 56 reference patients on conventional therapy. 26 patients achieved disease regression or remission ( $24 \mathrm{~h}$ proteinuria $<0.3$ g or $\geq 0.3$ and $<1$ g with improving or stable GFR, respectively) and were fully protected from cardiovascular events throughout the whole observation period. Again, treatment was well tolerated in all patients [7].
We, therefore, agree with Hirsch et al. [5] that 'initial creatinine increases over $30 \%$ should be tolerated when they occur in the context of goal-directed, more aggressive RAS inhibition' and that in this context 'guidelines that have aptly guided CKD care for over a decade should likely be updated'. Treatment should be individually tailored. An intensified approach targeting BP $\leq 125 / 75$ $\mathrm{mm} \mathrm{Hg}$ [4] and $24 \mathrm{~h}$ proteinuria $\leq 0.3 \mathrm{~g}$ [7] with maximum tolerated doses of ACEi and ARBs would be advisable in patients at risk of accelerated progression because of residual proteinuria despite conventional therapy. Conversely, single-drug RAS inhibition with recommended antihypertensive doses of an ACEi or an ARB might be the best option for those with no or subclinical proteinuria. Such patients normally are at low risk of progression and would therefore be unnecessarily exposed to the possible side effects of intensified RAS blockade without any appreciable benefit from proteinuria reduction [8]. Moreover, any progressive increase in serum creatinine that persists after 1-2 months of treatment should raise suspicion of a kidney hypoperfusion secondary to volume depletion and/or concomitant heart failure or kidney vascular disease. In such cases, treatment backtitration may be required to avoid consequences of chronic ischemic damage that might offset the benefits of decreased glomerular pressure and proteinuria.

Evidence is available that short-term GFR reduction predicts long-term renal function loss also in subjects with diabetes [9], including those without evidence of renal disease [10]. In a large cohort of type 2 diabetes patients prospectively monitored by serial direct GFR measurements over a median follow-up of 4 years, the GFR changes observed over the first 6 months of intensified metabolic BP control and ACEi therapy negatively correlated with subsequent GFR decline [10]. Again, subjects with more short-term GFR reduction were those with the larger benefit from intensified treatment in the long run. Of note, glomerular hyperfiltration, defined as a measured GFR $>120 \mathrm{ml} / \mathrm{min} / 1.73 \mathrm{~m}^{2}$, was an independent risk factor for renal disease onset and progression. In hyperfiltering subjects, a 6-month GFR reduction exceeding $10 \mathrm{ml} / \mathrm{min} / 1.73 \mathrm{~m}^{2}$ was associated with a protective effect against GFR decline and new onset of micro- or macroalbuminuria, whereas persistency of hyperfiltration despite intensified therapy invariably predicted poor outcomes [10].

Future studies should address whether and to what extent GFR reduction can be a specific treatment target for nephroprotection and hopefully identify novel and more effective interventions for those who respond poorly to 
intensified treatment. In every-day clinical practice, no patient with proteinuric CKD should be deprived of known strategies that delay progression and physicians should stay on course with a given therapy that effectively reduces BP and proteinuria independent of initial changes in serum creatinine. Sustained hyperkalemia and symptomatic hypotension should be the only stringent indications to treatment back-titration in this context [7].

\section{Acknowledgement}

Manuela Passera helped to prepare the manuscript.

\section{Disclosure Statement}

The authors have no conflicts of interest to disclose.

\section{References}

$>1$ Apperloo AJ, de Zeeuw D, de Jong PE: A short-term antihypertensive treatment-induced fall in glomerular filtration rate predicts long-term stability of renal function. Kidney Int 1997;51:793-797.

$\checkmark 2$ Anderson S, Brenner BM: Progressive renal disease: a disorder of adaptation. Q J Med 1989;70:185-189.

-3 Bakris GL, Weir MR: Angiotensin-converting enzyme inhibitor-associated elevations in serum creatinine: is this a cause for concern? Arch Intern Med 2000;160:685-693.

4 National Kidney Foundation I: Guideline 1: Goals of antihypertensive therapy in CKD. Am J Kidney Dis 2004;43:S183-S218.
5 Hirsch S, Hirsch J, Bhatt U, Rovin BH: Tolerating increases in the serum creatinine following aggressive treatment of chronic kidney disease, hypertension and proteinuria: pre-renal success. Am J Nephrol 2012;36 430-437.

-6 Vegter S, Perna A, Postma MJ, Navis G, Remuzzi G, Ruggenenti P: Sodium intake, ace inhibition, and progression to ESRD. J Am Soc Nephrol 2012;23:165-173.

$\checkmark 7$ Ruggenenti P, Perticucci E, Cravedi P, Gambara V, Costantini M, Sharma SK, Perna A Remuzzi G: Role of remission clinics in the longitudinal treatment of CKD. J Am Soc Nephrol 2008;19:1213-1224.
8 Ruggenenti P, Remuzzi G: Proteinuria: Is the ONTARGET renal substudy actually off target? Nat Rev Nephrol 2009;5:436-437.

$\checkmark 9$ Hansen HP, Rossing P, Tarnow L, Nielsen FS, Jensen BR, Parving HH: Increased glomerular filtration rate after withdrawal of longterm antihypertensive treatment in diabetic nephropathy. Kidney Int 1995;47:1726-1731.

10 Ruggenenti P, Porrini EL, Gaspari F, Motterlini N, Cannata A, Carrara F, Cella C, Ferrari S, Stucchi N, Parvanova A, Iliev I, Dodesini AR, Trevisan R, Bossi A, Zaletel J, Remuzzi G: Glomerular hyperfiltration and renal disease progression in type 2 diabetes. Diabetes Care 2012;35:2061-2068. 